\title{
Johannes Nollé
}

\section{Marktrechte außerhalb der Stadt: Lokale Autonomie zwischen Statthalter und Zentralort}

\section{Zur Fragestellung}

In den letzten Jahrzehnten wurde durch eine intensive Surveytätigkeit und zahlreiche Inschriftenfunde unser Wissen vom ländlichen Kleinasien in der Römischen Kaiserzeit erheblich erweitert. Wir kennen mittlerweile die Namen, oft auch die Lage von vielen ländlichen Gemeinden ${ }^{1}$; durch eine in vielerlei Hinsicht außergewöhnliche epigraphische Entdeckung verfügen wir sogar über eine vollständige Liste der Dörfer und Weiler eines Stadtterritoriums ${ }^{2}$; Inschriften und archäologische Überreste liefern uns eine schon nicht mehr zu überschauende Menge an Informationen, oftmals erstaunliche, aber meist aus den Zusammenhängen herausgerissene und der Interpretation bedürftige Details. Die neugewonnene Überlieferung lockt, eine Reihe von Fragen zu Administration, Wirtschaft, sozialen Verhältnissen, Religion und Kultur der kleinasiatischen Landgemeinden zu stellen und - zumindest in günstigen Fällen - auch Antworten zu versuchen. Meist aber sind die Ergebnisse dieser Erörterungen unsicher und haben vorläufigen Charakter. Das Bild des ländlichen Kleinasiens ist erst im Entstehen.

Ein zentrales Problem, das noch wenig diskutiert wurde, ist das der Spielräume ländlicher Selbstbestimmung. Ich gehe hier nicht auf das Problem jener ländlichen Siedlungen ein, die noch zu Beginn der Römischen Kaiserzeit als unabhängige administrative Einheiten in wenig urbanisierten Regionen neben den Städten standen, aber keinen Polis-Status besaßen. In der fortgeschrittenen Römischen Kaiserzeit dürfte durch die römische Urbanisierungspolitik ihre Zahl zurückgegangen $\operatorname{sein}^{3}$. Ich halte es für gut möglich, daß schließlich alle von ihnen einem bestimmten Stadtgebiet zugewiesen wurden, so daß Stephen Mitchell meiner Mei-

1 Sofern es sich dabei um einheimische Namen handelt, sind sie bei Ladislaus Zgusta, Kleinasiatische Ortsnamen (Heidelberg 1984) verzeichnet.

2 Michael Wörrle, Stadt und Fest im kaiserzeitlichen Kleinasien (München 1988) 135-150.

3 Die Meinung von David Magie, Roman Rule in Asia Minor (Princeton 1950) 1024: „The great majority of the known villages, however, seem not to have belonged to cities or temples but to have existed as separate rural communities" dürfte kaum zutreffend sein. 
nung nach richtig feststellt, Dorf sei in der hohen Kaiserzeit als „Gemeinwesen, das einer bestimmten Stadt untergeordnet war" definiert gewesen ${ }^{4}$. Die historische Entwicklung von ehemals zumindest nominell selbständigen Landgemeinden $\mathrm{zu}$ administrativen Einheiten eines Stadtterritoriums mag in der hohen Kaiserzeit insofern noch von Bedeutung gewesen sein, als die Erinnerung an ehemals autonome Dorf- bzw. Landgemeinden die Vorstellung von der Möglichkeit ländlicher Selbstverwaltung wachhielt. Aber auch abhängige Landgemeinden konnten über lokale Angelegenheiten eigene Entscheidungen treffen, wie den Quellen zu entnehmen ist. Unklar ist allerdings der Umfang ihrer Autonomie. Reichte sie bis zu Fällen, in denen bei dörflichen Angelegenheiten die römische Provinzadministration involviert war oder eingeschaltet werden mußte? Konnten Dörfer, die zum Territorium einer Stadt gehörten, in eigener Initiative und direkt, d.h. ohne Einschaltung des administrativ übergeordneten Zentralortes, mit der Spitze der römischen Provinzverwaltung, also mit dem Statthalter und seinem Officium, über lokale Angelegenheiten verhandeln wie auch Privilegien erwirken? War abhängigen Landgemeinden von ihrem Zentralort ein Selbstvertretungsrecht vor dem Statthalter zugestanden oder wurde wenigstens in Einzelfällen ein solches toleriert?

Es liegt auf der Hand, daß administrative Kontakte zwischen Landgemeinden und Statthalter - zwischen der höchsten und der untersten Organisationsebene einer Provinz - nicht besonders häufig vorkamen und daß sie nicht zum Alltag gehörten; dafür gab es zu viele psychologische, gesellschaftliche wie auch rechtliche Barrieren. Schon die Herstellung von Kontakten mit dieser Administrationsebene dürfte für Dorfleute in der Regel sehr schwierig gewesen sein. Es ist nicht zu erwarten, daß es in den Dörfern Männer gab, die Verbindungen zum Statthalter oder seiner Umgebung hatten; die regulären wie auch weniger offen zu Tage liegenden Wege, sich Zugang und Gehör zu verschaffen, dürften sie in der Regel nicht gekannt haben. Hemmungen und Ängste, aber auch mangelnde Fähigkeiten kamen hinzu: Wer traute sich und war von seiner Bildung her in der Lage, eine Petition zu überbringen, einen Antrag zu stellen oder gar Verhandlungen zu führen? Die Städte betrieben in der Römischen Kaiserzeit ihre Unterhandlungen mit den römischen Statthaltern meistens äußerst professionell; ihre Vertreter waren sehr oft Rhetoren, Sophisten und Rechtskundige ${ }^{5}$. Diese Standards waren für Landgemeinden schwer erreichbar' ${ }^{6}$. Außerdem mußten sie Verbote und Einschüchterun-

4 Stephen Mitchell, Anatolia. Land, Men, and Gods in Asia Minor 1 (Oxford 1993) 179: „One important consequence of this development was to define the status of a village, simply as a community subordinate to a particular polis."

5 Vgl. z.B. Glen W. Bowersock, Greek Sophists in the Roman Empire (Oxford 1969) $43 \mathrm{ff}$.; Angelos Chaniotis, Als die Diplomaten noch tanzten und sangen, in: ZPE 71 (1988) 154-156. ${ }^{6}$ Hier stellt sich grundsätzlich das Problem der ,Alphabetisierung' der Landbevölkerung; einige Überlegungen dazu in Johannes Nollé, Nundinas instituere et habere. Epigraphische Zeugnisse zur Einrichtung und Gestaltung von ländlichen Märkten in Afrika und in der Provinz Asia (Hildesheim, Zürich, New York 1982) $82 \mathrm{f}$. 
gen fürchten7: Ließen die Gesetze der Stadt, das Herkommen oder zumindest die städtischen Honoratioren den Dörfern überhaupt einen Spielraum? Und wie verhielt sich - vor allem im Konfliktfall - die römische Administration, die ihre Herrschaft auch durch Stützung oder zumindest Unterstützung der städtischen Honoratioren absicherte, zu solchen Aktivitäten der Dörfler ${ }^{8}$ ?

In ausweglos scheinenden Situationen, wenn die Bevölkerung eines Dorfes sich mit existentiellen Problemen konfrontiert glaubte und von der übergeordneten Stadt keine Hilfe zu erwarten hatte, überwanden Menschen aus abhängigen ländlichen Siedlungen die genannten Hindernisse. Wie uns die ,Hilferufe von Dörflern seit dem 2. Jahrhundert n. Chr. zeigen, wandten sie sich nicht nur direkt an den Statthalter ${ }^{10}$, sondern sogar an den Kaiser ${ }^{11}$. Diese Zeugnisse sind jedoch wenig hilfreich, das eingangs angesprochene Problem zu lösen, da sie Reflexe von Ausnahmesituationen sind, in denen oft die Verzweiflung Pate stand. Die Quellensituation für die Beantwortung der hier gestellten Frage ist nach Aussonderung dieser Zeugnisse ausgesprochen schlecht. Allerdings sind in der Provinz Asia Dokumente ans Tageslicht gekommen, denen bei einer genaueren Betrachtung doch einige Erkenntnisse zu dieser Frage abzuringen sind; dabei handelt es sich um vier

7 Vgl. z.B. Mitchell, Anatolia (Anm. 4) 194: „cities in general sought to control and exploit their rural territories".

8 Vgl. dazu etwa die Überlegungen von Richard Klein, Die Romrede des Aelius Aristides. Einführung (Darmstadt 1981) 68; Friedrich Quaß, Die Honoratiorenschicht in den Städten des griechischen Ostens. Untersuchungen zur politischen und sozialen Entwicklung in hel-

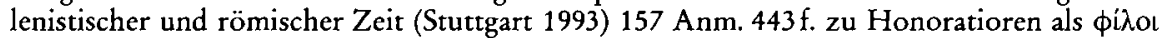
des Statthalters. Besonders aufschlußreich ist Hans Joachim Gehrke, Thisbe in Boiotien. Eine Fallstudic zum Thema „Griechische Polis und Römisches Imperium“, in: Klio 75 (1993) 145154, bes. 154: „Die wirtschaftliche Struktur auch in den kleinsten Bereichen fand das Interesse der imperialen Zentrale; diese bemühte sich durchaus um eine patronale Fürsorge für die Gemeinde, setzte aber dabei just auf die ökonomischen Interessen der Honoratiorenschicht. Damit stellte sie unter Beweis, wie selbstverständlich die Interessen von Polis und Plusioi unter dem Schutz der römischen Verwaltung zusammenfallen konnten. “

$9 \mathrm{Zu}$ dem Konflikt zwischen Dorf und übergeordneter Stadt vgl. z. B. Mitchell, Anatolia (Anm. 4) $195 \mathrm{f}$.

$10 \mathrm{Vgl}$. die Petition von Dorfbewohnern aus Choradaga auf dem Territorium von Histria in der Moesia Inferior an den Statthalter (SEG XIX 476; Dionisie M. Pippidi, Inscriptiones Scythiae Minoris Graecae et Latinae I: Inscriptiones Histriae et viciniae [Bukarest 1983] 490 Nr. 378).

11 Vgl. etwa Fergus Millar, The Emperor in the Roman World (31 BC - AD 337) (London 1977) 541 f.; Frank Kolb, Die Stadt im Altertum (München 1984) 256: „Und obwohl die AuBenbeziehungen des Territoriums zum Provinzstatthalter und zum Kaiser grundsätzlich über die munizipalen Behörden liefen, konnten Dörfer, die sich ungerecht behandelt fühlten, doch unmittelbar mit der Zentralgewalt Kontakt aufnehmen“; Walter Scheidel, Dokument und Kontext: Aspekte der historischen Interpretation epigraphischer Quellen am Beispiel der „Krise des dritten Jahrhunderts“, in: RSA 21 (1991) 145-164, bes. 156f.: „Direkte Eingaben an den fernen Kaiser, für städtische Gemeinwesen von Anfang an bekanntlich nichts Ungewöhnliches, mochten für provinziale Bevölkerungsgruppen im ländlichen Raum lange Zeit keine realistische Option dargestellt haben“. Er sieht die „längerfristige Akkulturation der provinzialen Landbevölkerung" als eine wichtige Vorbedingung für die direkten Petitionen an. 
inschriftlich überlieferte Dokumente bzw. Dokumentenzusammenstellungen, die mit der Verleihung von Marktrechten an Dörfer zu tun haben.

\section{Zur Entwicklung der Dörfer in der Provinz Asia während der Kaiserzeit}

Die Römische Kaiserzeit wurde bis etwa in die ersten Jahrzehnte des 3. Jahrhunderts n. Chr. von großen Teilen der Landbevölkerung der Provinz Asia trotz verschiedenster Probleme wahrscheinlich als eine durchaus erträgliche, wenn nicht gar gute Zeit empfunden. Die Pax Romana, die äußere und auch innere Sicherheit schuf, bot die Voraussetzung für eine weitgehend ungestörte wirtschaftliche Aufwärtsentwicklung und für eine spürbare Steigerung der Lebensqualität auf dem Lande. Anscheinend erreichten zahlreiche Dörfer in jenen Regionen Kleinasiens, wo es viel fruchtbares Land gab und in größerem Umfang agrarische Überschüsse erzielt werden konnten, einen erkennbaren Wohlstand. Sowohl die Idealisierung des Landlebens als auch die Dramatisierung von Leiden der auf dem Lande lebenden unterdrückten Menschen sind Extreme, die in Einzelfällen und bestimmten Situationen der Realität nahekommen können, nicht aber den allgemeinen $\mathrm{Zu}$ stand richtig wiedergeben.

Die Auswirkungen der positiven Entwicklung der ökonomischen Verhältnisse sind vor allem in Inschriften aus dem ländlichen Raum gut faßbar. Die Nachahmung urbaner Organisationsformen und Versuche, sich städtischen Standards anzunähern, sind nicht zu übersehen ${ }^{12}$. Das betrifft einerseits die politische Struktur der Dörfer, andererseits die Bautätigkeit, die für größere ländliche Siedlungen bei günstiger Überlieferung inschriftlich ansatzweise faßbar ist. Das Bestreben der Dörfler, nach städtischem Vorbild ein politisches und wirtschaftliches, baulich gestaltetes Zentrum im ländlichen Raum zu konstituieren und in diesem ruralen Zentralort in städtischen Formen zu agieren ${ }^{13}$, wird erkennbar. Da es umgekehrt Siedlungen gab, die Stadtrecht besaßen, durch ihre bauliche Ausgestaltung aber nur wenig oder überhaupt nicht urban geprägt waren ${ }^{14}$, begannen gerade die äußeren, d.h. sichtbaren Unterschiede zwischen schlechtentwickelter Stadt und hochentwickeltem Dorf $z u$ verschwimmen, wurden unscharf und vage ${ }^{15}$.

12 Zu diesem Phänomen vgl. Cesare Letta, L'epigrafia pubblica di vici e pagi nella Regio IV: Imitazione del modello urbano e pecularità del villagio, in: Alda Calbi, Angela Donati, Gabriella Poma, L'epigrafia del villaggio (Faenza 1993) 33-48.

13 Aufschlußreich sind in dieser Hinsicht die Beobachtungen von Michael Wörrle, Antiochos I., Achaios der Ältere und die Galater. Eine neue Inschrift in Denizli, in: Chiron 5 (1975) 59-87, bes. 85 zu zwei hellenistischen Dörfern, die dörfliche Einrichtungen mit städtischen Termini belegen.

14 Das Standardbeispiel ist die phokische Polis der Panopeis (Pausanias 10, 4, 1), die weder Amtsgebäude noch Gymnasium, Theater und Agora besaß.

15 Vgl. z. B. Mitchell, Anatolia (Anm. 4) 181 zu den Verhältnissen in Kleinasien: „Only an arbitrary decision about status, designed for administrative convenience, will have ordained a 
Der Aufmerksamkeit der Zeitgenossen sind diese Entwicklungen nicht entgangen; so ist bei dem Grammatiker Festus aus dem 2. Jahrhundert n. Chr, zu lesen, daß einige Dörfer eine regelrechte Verfassung hätten: sed ex vicis partim habent rempublicam et ius dicitur, ... et magistri vici, item magistri pagi quotannis fiunt ${ }^{16}$. Tatsächlich sind uns dörfliche ,Magistrate (Pseudo-Magistrate ?) unter vielerlei Namen für zahlreiche Aufgabenbereiche in Dörfern bezeugt. Einige von ihnen zahlten wie städtische Amtsträger ,summae honorariae' für ihre Ämter ${ }^{17}$; nach ihnen wurde datiert, sie waren ,eponyme Beamte ${ }^{18}$. Gar nicht selten hören wir auch von der politisch agierenden Dorfgemeinde, die in der Kaiserzeit meist als

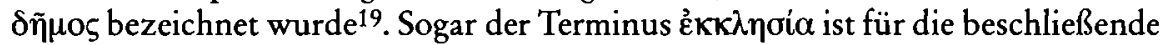
Dorfversammlung bezeugt ${ }^{20}$; Dörfer verabschiedeten $\psi \eta \phi i \sigma \mu \alpha \tau \alpha^{21}$. Selbst ein

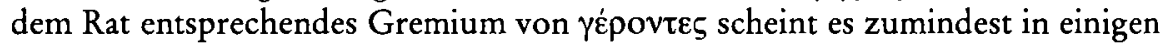
Dörfern gegeben zu haben ${ }^{22}$. Ob allerdings die von Karl Buresch getroffene Fest-

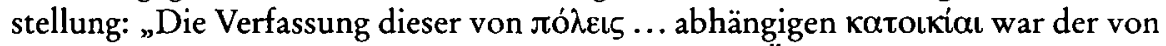
selbständigen Städten genau nachgebildet " durch die Überlieferung abgedeckt ist, wage ich zu bezweifeln ${ }^{23}$.

Religiöse Feste auf dem Lande wurden wie in den Städten mit der Austragung von Agonen begangen; sogar Gymnasien wurden auf dem Lande etabliert, wo die ,Landjugend" städtischen Schliff erhalten sollte ${ }^{24}$. In vielen Dörfern wurden Bäder gebaut $^{25}$; es ist bezeichnend, daß gerade Thermen in den Augen der antiken Men-

difference between katoikiai like Lyendos, Ariandos, Apateira, and the Teirenon katoikia and poleis like Bagis, Maeonia, Tmolus, and Hypaepa."

16 Festus bei Paulus Diaconus (ed. Lindsay) 502, Z.13ff.

17 Vgl. G. M. Harrer, Village Administration in Roman Syria, in: YCS 1 (1928) $142 \mathrm{ff}$.

18 Z.B. in dem Marktdokument von Mandragoreis vgl. Nollé, Nundinas (Anm. 6) 13 Z. 48f.; ferner die Petition der Dorfbewohner von Choradaga (vgl. Anm. 10) Z. 21 ff.; vgl. auch Letta, Regio IV (Anm. 12) 41.

19 Dazu Wörrle, Antiochos (Anm. 13) 85f. Anm. 132; Georg Petzl, H. W. Pleket, Inschriften aus Lydien, in: ZPE 34 (1979) 281-295, h. 287; Wolfgang Leschborn, Mysomakedones, in: JNG 34 (1984) 55-62, bes. 62; Michael Wörrle, Inschriften von Herakleia am Latmos I: Antiochos III, Zeuxis und Herakleia, in: Chiron 18 (1988) 421-476, bes. 469; Thomas DrewBear, George Le Rider, Monnayage cistophorique des Apaméens, des Praipénisseis et des Corpéni sous les Attalides. Questions de géographie historique, in: BCH 106 (1991) 361-376, h. 372 in Phrygien.

20 TAM V 1, 222; vgl. dazu Wörrle, Antiochos (Anm. 13) 85 mit Anm. 134.

21 Z.B. TAM V 1, 228 und 234.

22 TAM V 1, 222; vgl. auch W. H. C. Frend, A Third-Century Inscription Relating to Angareia in Phrygia, in: JRS 56 (1956) 46-56, bes. 53. Der sichere Nachweis einer dörflichen Bule scheint mir trotz verschiedener Hinweise für Kleinasien noch nicht geglückt zu sein; vgl. etwa Karl Buresch, Aus Lydien. Epigraphisch-geographische Reisefrüchte (Leipzig 1898) 2; Wörrle, Antiochos (Anm. 13) 85 Anm. 132 mit Verweis auf Buresch; Maurice Sartre, Communautés villageoises et structures sociales d'après l'épigraphie de la Syrie du Sud, in: Alda Calbi, Angela Donati, Gabriella Poma, L'epigrafia del villaggio (Faenza 1993) 117-135, bes. 121 f.; Mitchell, Anatolia (Anm. 4) 180 Anm. 137.

23 Buresch, Lydien (Anm. 22) 2.

24 Vgl. etwa Mitchell, Anatolia (Anm. 4) 187.

${ }^{25}$ L. de Ligt, Demand, Supply, Distribution: The Roman Peasantry between Town and Countryside: Rural Monetarization and Peasant Demand, in: Münstersche Beiträge zur anti- 
schen als Indiz für städtisches Leben und urbanes Niveau galten ${ }^{26}$. Ein Dorf auf dem Territorium des lydischen Philadelpheia legte sogar eine Agora an und bekundete dies durch eine beschriftete Säule, die auf diesem Marktplatz aufgestellt wurde ${ }^{27}$. Wir wissen zwar nichts über Art und Umfang des Warenaustauschs, der auf diesem dörflichen Marktplatz stattfand, aber im günstigsten Fall ist ein solches Dorf auf dem Weg, nach Max Webers Definition ,Stadt im ökonomischen Sinnec zu werden ${ }^{28}$. Das gilt besonders für jene Dörfer, die nicht nur eine Agora bauten, sondern die für diese auch Marktrechte gewinnen konnten. Selbstverständlich ist die Einschätzung Max Webers nicht die der antiken Menschen, für die die Bezeichnung,Stadt' politisch-administrativ, wenn wir so wollen, rechtlich definiert war, für die die wirtschaftliche Ausstattung einer Siedlung allenfalls eine knappe Notiz, ein schmückendes Epitheton wert war. Nur in Ausnahmefällen war es für eine ländliche Siedlung möglich, aufgrund einer enormen Verbesserung der wirtschaftlichen und politischen Infrastruktur zu einer selbständigen Stadt aufzusteigen und sich aus dem Territorium und der Verfügungsgewalt des übergeordneten Zentralortes herauszulösen. Wahrscheinlich ging es den meisten Menschen auf dem Lande nicht um das Erreichen solch hochgesteckter Ziele. Die Fortschritte mancher ländlichen Siedlungen in der Römischen Kaiserzeit führten eher, und daran kann kein Zweifel sein, zu wachsendem Selbstbewußtsein der Menschen, die dort lebten. Die in der hohen Kaiserzeit stark anwachsende Zahl von Inschriften auf dem Lande ist nicht zuletzt Ausdruck des Bestrebens, Erfolge der ländlichen Gemeinschaften nach städtischem Muster bekannt zu machen und eine eigene, lokale Identität herauszustellen. Dieses veränderte Bewußtsein und möglicherweise eine verstärkte Wertschätzung des eigenen Lebensraumes und seiner tradierten Regeln wird die Frage nach Freiheiten und Spielraum für eine Eigenbestimmung aufgebracht haben. Gerade die Vorgänge um die Gewinnung eines

ken Handelsgeschichte 9 (1990) 24-30, h. 28 f. mit Anm. 17 zu den Verhältnissen in Gallien. $\mathrm{Zu}$ ländlichen Thermen vgl. z. B. Louis Robert, Sur un papyrus de Bruxelles, in: RPh 17 (1943) 111-119, bes. 115f. (= ders., Opera Minora Selecta 1 [Amsterdam 1969] 364-372, bes. $368 \mathrm{f}$.) zu einem Dorfbad, das aus Not aufgegeben werden mußte; ders., Hellenica 9 (Paris 1950) 30 mit Anm. 5; H. W. Pleket, Nine Greek Inscriptions from the Cayster-valley in Lydia: A Republication, in: Talanta 2 (1970) 55-88, bes. $80 \mathrm{f}$. mit weiteren Literaturhinweisen; Peter Herrmann, Zur Geschichte der Stadt Iulia Gordos in Lydien (Öst. Akad. Wiss., Phil.-hist. Kl., 107, 1970) Nr. 6, 92-103, bes. $100 \mathrm{ff}$.

26 Es ist bezeichnend, daß Orkistos (I $25 \mathrm{f}$.) als Beweis für seine städtische Infrastruktur u.a. anführt: labacra quoque publica privataque; vgl. dazu etwa André Chastagnol, L'inscription constantinienne d'Orcistus, in: MEFRA 93 (1981) 381-416, bes. 406.

27 Georg Petzl, Epigraphische Funde aus Lydien, in: EA 15 (1990) 49-72, h. 70 Nr. 34: ětous

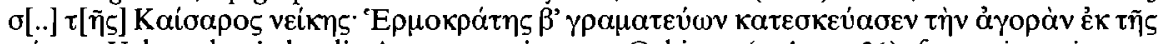

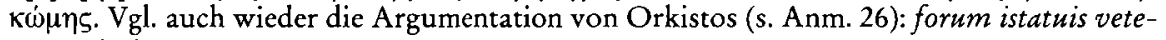
rum principum ornatum.

28 Max Weber, Wirtschaft und Gesellschaft: Grundriß der verstehenden Soziologie (Tübingen 51976) 728: „Wir wollen von ,Stadt ${ }^{\circ}$ im ökonomischen Sinn erst da sprechen, wo die ortsansässige Bevölkerung einen ökonomisch wesentlichen Teil ihres Alltagsbedarfs auf dem örtlichen Markt befriedigt, und zwar zu einem wesentlichen Teil durch Erzeugnisse, welche die ortsansässige und die Bevölkerung des nächsten Umlandes für den Absatz auf dem Markt erzeugt oder sonst erworben hat." 
durchaus substantiellen Rechts wie des Marktrechts können somit zur Erhellung der Problematik, Lokale Autonomie` Wesentliches beitragen.

\section{Inschriften über Marktrechtsverleihungen und ihr Zeugniswert}

Inzwischen liegen uns drei bzw. vier inschriftliche Zeugnisse aus der Provinz Asia vor, die Informationen darüber liefern, wie ländliche Siedlungen Marktrecht gewannen. Hasan Malay hat vor kurzem eine Stele aus dem Territorium von Sardeis bekannt gemacht, die uns über die Einrichtung eines Jahrmarktes in der Landgemeinde der Arillenoi auf dem Territorium von Sardeis in der Zeit um $136 \mathrm{n}$. Chr. unterrichtet ${ }^{29}$; Werner Eck und ich haben ein auf dieser Stele wiedergegebenes, fragmentarisch erhaltenes Dossier des Textes neu ergänzt und seinen Sinn erhellt ${ }^{30}$. Vor einigen Jahren habe ich eine Inschrift publiziert, die uns über die Institutionalisierung eines Dekadenmarktes ${ }^{31}$ im Dorf Mandragoreis auf dem Territorium von Magnesia am Mäander im Jahre $209 \mathrm{n}$. Chr. informiert ${ }^{32}$. Schon lange bekannt ist eine Inschrift aus der Kastolosebene in Lydien, die das Privileg für einen monatlichen Markttag zugunsten des Dorfes Tetrapyrgia aus dem Jahre 253/4 n. Chr. wiedergibt ${ }^{33}$. Die Dokumente stammen also aus ganz unterschiedlichen Zeiten und beziehen sich auf verschiedenartige Marktrechte: Jahrmarkt, Monatsmarkt und Dekadenmarkt. In die Diskussion einzubeziehen ist schließlich noch ein spätes Dokument - es stammt vermutlich aus dem späteren 3. oder beginnenden 4. Jahrhundert ${ }^{34}$-, in dem es um die Verleihung von Privilegien für das Dorf der Pyliten geht, das wahrscheinlich auf dem Territorium von Magnesia am Mäander oder auf dem der Nachbarstadt Tralleis lag 35 . Die Art der Privilegien für die Pyliten kennen wir nicht, da eine Stele, auf der sie verzeichnet waren, verlorengegangen ist. Die erhaltene zweite Stele gibt nur Auskunft über den Verfahrensweg, der zur Gewährung der Privilegien führte. Es ist gut möglich, ich meine, sogar

${ }^{29}$ Hasan Malay, Greek and Latin Inscriptions in the Manisa Museum (Wien 1994) 152-156 Nr. 523.

30 Der Brief des Asinius Rufus an die Magistrate von Sardeis. Zum Marktrechtsprivileg für die Gemeinde der Arillenoi, in: Chiron 26 (1996) 267-273.

31 D.h. es wird in jeder der drei Dekaden eines Monats Markt abgehalten.

32 Nollé, Nundinas (Anm. 6) 11-58; SEG 32 (1982) 331 ff., Nr. 122.

33 TAM V 1, 230; vgl. Nollé, Nundinas (Anm. 6) 59-86; SEG 32 (1982) 331 ff., Nr. 1220.

34 Möglicherweise ist der darin genannte Proconsul Taurus mit Flavius Taurus, dem späteren Praefectus praetorio Occidentis Italiae et Africae (355-361) des Constantius zu identifizieren (PLRE I, s.v. Flavius Taurus 3, $879 \mathrm{f}$.; Raban von Haebling, Die Religionszugehörigkeit der hohen Amtsträger des Römischen Reiches seit Constantins I. Alleinherrschaft bis zum Ende der Theodosianischen Dynastie [Bonn 1978] 293 f.).

35 Hasan Malay, Letter of the proconsul Taurus and the People of Pylitae near Tralles, in: EA 11 (1988) 53-58; Jobannes Nollé, Epigraphische und numismatische Notizen 9: Zu der neuen Stele aus dem Museum von Aydın, in: EA 15 (1990) 121-125. Vgl. Denis Feissel, REG 105 (1992) 504 f. Nr. 432; SEG 38 (1988) 349 Nr. 1172. 
wahrscheinlich, daß die Rechtsgewährung, um die es hier geht, die Ausstattung eines ländlichen (natürlich religiösen) Festes mit Marktrecht beinhaltete.

Aus den vier Stelen - die aus einem Zeitraum von ca. 200 Jahren stammen und keinen Eindruck von der Häufigkeit solcher Privilegierungen geben, allenfalls zu dem voreiligen Schluß verführen könnten, daß sie selten vorkamen -, erfahren wir, daß derartige Rechtsverleihungen keine Einzelfälle waren: In dem Marktprivileg aus dem Stadtgebiet von Sardeis heißt es, daß die Stadt Sardeis im Begriff war, sich um den Ausbau der Privilegien von Dörfern ihrer Chora, die schon das Markt-

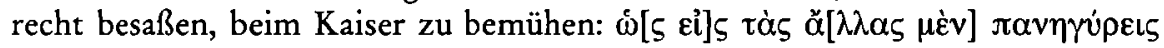
$\pi \varepsilon \dot{v} \pi \varepsilon \tau \varepsilon$, d. h. daß Ibr Euch um die anderen Jabrmärkte (nämlich die Jahrmärkte anderer Dörfer) mit Gesandtschaften bemüht. In der Inschrift von Mandragoreis argumentiert der uns nicht bekannte Antragsteller damit, daß schon eine Reihe von anderen Dörfern vor Mandragoreis mit Marktrecht ausgestattet wurden:

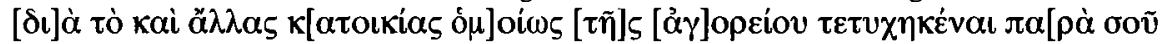

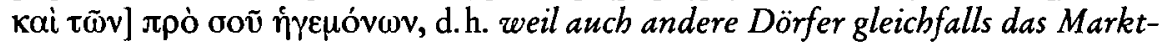
recht von Dir oder Deinen Vorgängern im Statthalteramt erbalten baben. In der späten, aus dem Jahr $254 \mathrm{n}$. Chr. stammenden Marktrechtsverleihung für Tetrapyrgia wird darauf hingewiesen, daß vor Tetrapyrgia schon andere Dörfer auf dem Stadtgebiet von Maionia einen Markttag einrichten konnten, was uns nach den beiden früheren Dokumenten aus der hadrianischen und der severischen Zeit nicht wundern kann. Wir dürfen also festhalten, daß im Laufe des 2. Jahrhunderts und noch im 3. Jahrhundert die Einrichtung von ländlichen Märkten in der Provinz Asia kein seltenes Phänomen war. Wir gewinnen demnach durch die wenigen erhaltenen Dokumente einen Hinweis auf ein häufiges, wenn nicht sogar typisches Phänomen dörflicher Entwicklung in der Römischen Kaiserzeit ${ }^{36}$. Auch im übrigen Reich scheint die Ausstattung einer Landgemeinde mit irgendwelchen Marktrechten nicht unüblich gewesen zu sein: In dem bereits zitierten Festus-Lexikon wird unter dem Stichwort ,Dorf' als bemerkenswerte Besonderheit von Dörfern nicht nur die Existenz von dörflichen Magistraten angesprochen, sondern sind auch Märkte genannt: et tamen ibi nundinae aguntur negoti gerendi causa ${ }^{37}$. Wir müssen deshalb folgern, daß wir es mit einer ausgesprochen schlechten Überlieferung für ein häufiges Phänomen zu tun haben. Das mag einerseits mit einer hohen Verlustrate gerade bei großen und empfindlichen Inschriftenträgern wie Stelen zusammenhängen ${ }^{38}$; zum anderen könnte aber die aufwendige

36 Auf die Konsequenzen, die sich daraus für die Einschätzung der antiken Wirtschaft als ,primitiv" und ,nicht marktorientiert' ergeben, ist bereits $L$. de Ligt, Fairs and Markets in the Roman Empire. Economic and Social Aspects of Periodic Trade in a Pre-Industrial Society (Amsterdam 1993) 91-105 ausführlich eingegangen.

$37 \mathrm{Vgl}$. Anm. 16.

38 Bezeichnenderweise ist von dem Edikt des Sex. Sotidius Strabo Libuscidianus (Stephen Mitchell, Requisitioned Transport in the Roman Empire: A New Inscription from Pisidia, in: JRS 66 [1976] 106-131), das, wie es im Text heißt, in der Stadt und in jedem Dorf publiziert werden sollte (formulam eorum quae [prae]stari iudico oportere in singulis civitatibus et vicis

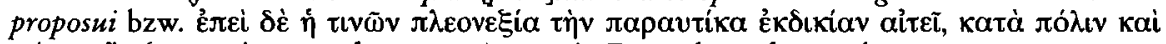

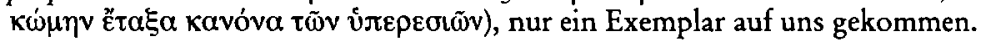


Errichtung einer Stele, die den Text der gewährten Privilegien trug, die Ausnahme gewesen sein; vielleicht mußten, gerade im dörflichen Zusammenhang, erst besondere Umstände eintreten, ehe es zur Anfertigung und Aufstellung von solchen Monumenten kam.

Die wenigen erhaltenen Inschriften gewähren uns detaillierte Einblicke in die Vorgänge, wie Marktrechte für Dörfer durch das Zusammenspiel mehrerer Interessensgruppen erreicht wurden. Obwohl es dabei immer darum ging, daß der Proconsul der Provinz das Privileg gewährte, werden bei genauerer Betrachtung Unterschiede deutlich, die vor allzu schnellen Verallgemeinerungen und zu weit gehenden Schlußfolgerungen warnen. Offensichtlich gab es keinen fest institutionalisierten, peinlich genau einzuhaltenden Verfahrensweg. Außerdem ist die Verteilung der Dokumente auf einen langen Zeitraum - von ca. $136 \mathrm{n}$. Chr. bis zum Ende des 3. bzw. Anfang des 4. Jahrhunderts n. Chr. - und zwei geographisch verschiedene Räume - das untere Mäandertal und Lydien - zu bedenken. Es ist mit administrativen Veränderungen, Entwicklungen in der Infrastruktur sowie auch mit lokalen Eigenheiten zu rechnen. Stephen Mitchell hat im Rahmen seines Buches ,Anatolia' in dem Kapitel über das Landleben darauf aufmerksam gemacht, daß gerade die Verhältnisse in Lydien, wo zwei der hier zu behandelnden Dokumente herkommen, nicht typisch für das übrige Kleinasien sein müssen ${ }^{39}$.

\section{Initiativen von Landgemeinden und die römische Administration}

Eine wichtige Voraussetzung für lokale Autonomie waren Eigeninitiative und Engagement von Menschen der dörflichen Gemeinden. Läßt sich diese auch bei der Gewinnung von Marktrechten feststellen? Gingen die Bestrebungen, solche Rechte zu gewinnen, von den Landgemeinden selber aus, oder waren eher die Interessen von höher stehenden Einzelpersonen oder von Städten, solche Märkte zu etablieren, konstitutiv? Besonders aufschlußreich für diese Fragestellung ist die neue Marktstele aus dem Stadtgebiet von Sardeis. Treibende Kraft bei der Gewinnung des Jahrmarktes war ein gewisser Metrâs, der sich als Priester auf Lebenszeit

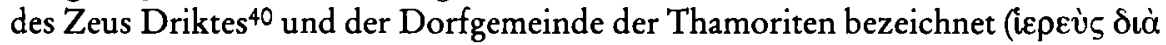

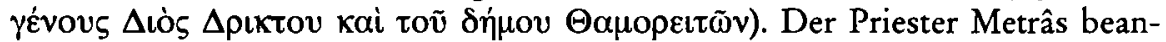

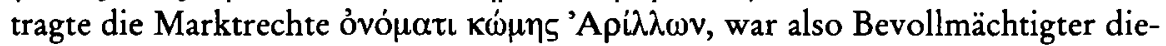
ser Landgemeinde, und der Proconsul gewährte sie auch ausdrücklich den Arillenoi. Die nach der Verleihung des Privilegs gefertigte Stele mit den Texten über die Marktrechtsgewährung an die Arillenoi weihte Metrâs in Erfüllung eines Gelüb-

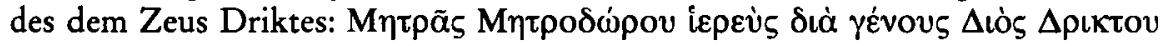

39 Mitchell, Anatolia (Anm. 4) $181 \mathrm{f}$.

40 Diese Namensform ergibt sich aus einer Weihung für ihn: Malay, Manisa Museum (Anm. 29) 48 Nr. 56. 


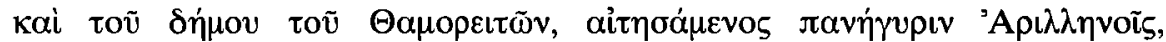

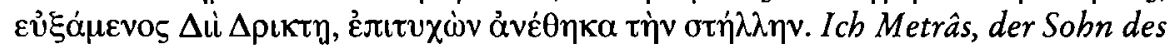
Metrodoros, Priester in Familienerbfolge des Zeus Driktes und des Dorfes der Thamoriten, stellte für die Arillenoi den Antrag auf einen Jabrmarkt, legte vor Zeus Driktes ein Gelïbde ab, wurde erbört und weibte die Stele. Aus dem Wortlaut der Dedikationsinschrift möchte ich schließen, daß die Stele als Votivgabe bei dem Heiligtum dieses Gottes aufgestellt wurde. Sie erfüllte dort noch eine weitere Funktion: Die ausführliche, für ein Votiv überflüssige Wiedergabe der Marktprivilegien in lateinischer und griechischer Sprache macht dann am ehesten Sinn, wenn der Aufstellungsort identisch mit dem Marktort war. Das Heiligtum des Zeus Driktes dürfte also in der Dorfgemeinde der Arillenoi gelegen haben; bei ihm fand alljährlich im Herbst, in der Zeit vom 20.-26. September, der vom Proconsul genehmigte Jahrmarkt statt.

Metrâs engagierte sich demnach in seiner Eigenschaft als Priester des Zeus Driktes für die Marktprivilegien zugunsten des Dorfes der Arillenoi und des Heiligtums. Wahrscheinlich sollte durch die Abhaltung von Markttagen ein lokal, vielleicht sogar regional wichtiges ${ }^{41}$ religiöses Fest ${ }^{42}$ zu Ehren des Zeus Driktes noch attraktiver gestaltet werden. Die Zentralort-Funktion des ländlichen Heiligtums wurde durch die Etablierung des Jahrmarktes gestärkt.

Mit der Publikation des Marktrechtes auf einer dem Zeus Driktes geweihten Stele bei dessen Tempelbezirk wird die Gewinnung des Privilegs wie auch die Abhaltung des Marktes in einen religiösen Kontext gestellt. Es ist vor allem der heimatliche Gott, dem die Gewährung des Marktrechtes zu danken ist ${ }^{43}$; der Priester Metrâs und der römische Proconsul mußten den Gläubigen in letzter Konsequenz als Werkzeuge in der Hand des Gottes erscheinen. Ein Festmarkt mit der Möglichkeit, auf ein breiteres Warenangebot als üblich zurückgreifen zu können, steigerte nicht nur die Festesfreude der Teilnehmer, sondern wies auch die Gottheit als Spender der neuen Wohltat aus und festigte die Dankbarkeit ge-

41 Auf die regionale Bedeutung ländlicher religiöser Zentren geht Mitchell, Anatolia (Anm. 4) 185 ff. ein.

42 Vgl. das jährliche Fest der Dörfergruppe von Ihsaniye auf dem Territorium von Nikomedeia (TAM IV 1, Nr. 15-18 und dazu den Kommentar von Louis Robert, Voyages épigraphiques en Asie Mineure, in: RPh 17 [1943] 170-201, bes. 189-194).

43 Ähnliche Vorstellungen finden wir auch in der hellenistischen Privilegierung für Baitokaike (J.-P. Rey-Coquais, IGLS VII 4028; vgl. J.-P. Rey-Cocquais, Des montagnes au désert: Baetocécé, les pagus Augustus de Niha, la Ghouta et l'est de Damas, in: E. Frézouls [Hrsg.], Sociétés urbaines, sociétés rurales dans l'Asie Mineure et la Syrie hellénistiques et romaines. Actes du colloque de Strasbourg, novembre 1985 [Strasbourg 1987] 191-216; A. Baroni, I terreni e i privilegi del tempio di Zeus a Baitokaike [Inscriptiones Graece et Latinae Selectae VII, 4028], in: $B$. Virgilio [Hrsg.], Studi Ellenistici 2 [Pisa 1987] 135-167), bei der der seleukidische

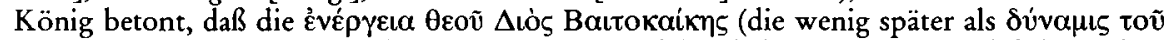
$0 \varepsilon 0 \tilde{v}$ bezeichnet wird) zu der Privilegierung geführt habe. In einer nordafrikanischen Inschrift werden ausdrücklich Götter mit der Einrichtung eines Jahrmarktes in Verbindung gebracht: nundina annu(a), quod praecepit Iovis et Iuba et Genius Vanisne(n)si(um), quod pr(a)eceperunt dii Ingirozogle(n)zi(u)m (CIL VIII 20.627; vgl. Nollé, Nundinas [Anm. 6] $154 \mathrm{f}$.$) .$ 
genüber ihrem Priester. Die Gewinnung von Marktrechten für ein religiöses Fest dürfte vorzüglich geeignet gewesen sein, die Bindungen der ländlichen Bevölkerung an ihre lokalen Gottheiten zu verstärken, den Menschen auf dem Lande Vertrauen in ihre religiösen Führer zu vermitteln und eine lokale Identität zu schaffen.

Darüberhinaus werden noch andere Überlegungen den Priester Metrâs zu seinem Engagement für den Jahrmarkt bewogen haben. Wirtschaftliche Vorteile liegen auf der Hand. Es ist aber auch daran zu denken, daß bereits erfolgte Verleihungen von Marktrechten an andere Landgemeinden Sorge bei den Arillenoi aufkommen ließen, sie könnten hinter die Ausstattung anderer Dörfer bzw. anderer Heiligtümer der Region zurückfallen. Dabei dürfte es nicht nur um ,Nachahmungstrieb' gegangen $\operatorname{sein}^{44}$, sondern auch um Rivalität ${ }^{45}$. Hinter dem Hinweis auf die Präzedenzfälle bei der Verleihung von Marktrechten in dem einen Dokument aus Mandragoreis dürften auch derartige Erwägungen bzw. Befürchtungen gestanden haben 46 .

Eine Vielzahl von Motiven: Frömmigkeit, persönlicher Ehrgeiz, lokales Prestigedenken und wirtschaftliche Erwägungen, brachten Metrâs dazu, sich um Marktrechte für das Dorf der Arillenoi zu bemühen. Metrâs - in Begleitung eines gewissen Isidoros, den man am ehesten als einen ,Magistraten' der Kome der Arillenoi identifizieren möchte - wurden persönlich bei dem Proconsul der Provinz Asia, T. Aurelius Fulvus Boionius Antoninus, dem späteren Kaiser Antoninus Pius, vorstellig und beantragten das dörfliche Marktrecht. Aus der Formulierung qua de re si quis petitioni eius contradicere volet, intra diem tricensimum me aut successorem meum c. v. adeat ist zu schließen, daß die Verhandlung in den letzten Tagen seiner Statthalterschaft stattgefunden haben muß. Mit der von ihm gewählten Formulierung weist der Proconsul darauf hin, daß die 30 Tage der eingeräumten Widerspruchsfrist gegen die Einrichtung des Jahrmarktes nicht mehr vollständig in seine Amtszeit fallen ${ }^{47}$. Vermutlich hielt er sich in den letzten Tagen seiner Amtszeit in Ephesos auf.

In dem Edikt des Proconsuls sind die beiden dörflichen Gesandten namentlich erwähnt, was sie sicherlich mit Stolz erfüllt hat: T. Aurelius Ful(v)us Boeonius Antoninus procos. dic.: Metras Metrodori et Isidorus Isidori nom. vicanorum Arbillon adierunt me petieruntque uti eis permitterem mercatum instituere et habere semel in anno diebus continuis septem ex XII $k$. Octobr. in VI k. easdem. T. Aủp

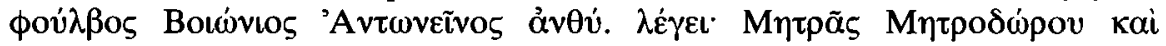

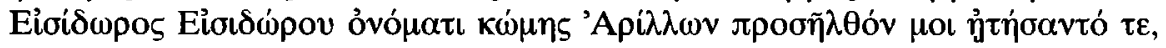

44 Von ,Nachahmungstrieb‘ spricht Scbeidel, Dokument (Anm. 11) 159.

45 Auf die gefährdete Eintracht der Dörfer weist die ländliche Weihung einer Statue der ,Homonoia der Dörfer' aus dem Stadtgebiet von Nakoleia (Thomas Drew-Bear, Nouvelles inscriptions de la Phrygie [Zutphen 1978] 50 Nr. 26; vgl. A. R. R. Sheppard, Homonoia in the Greek Cities of the Roman Empire, in: AncSoc 16-17 [1984-86] 229-252, h. 231).

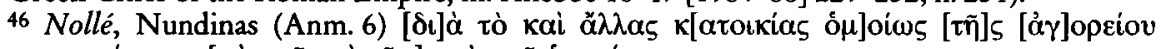

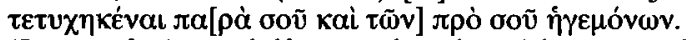

47 Darauf wies Rudolf Haensch in der Diskussion während des Kolloquiums hin. 


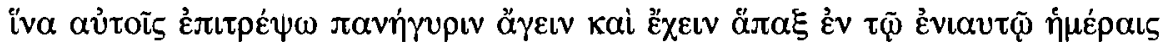

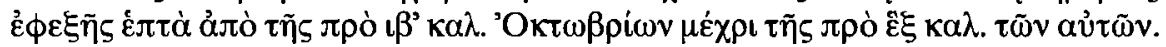
Engagement und Unternehmungsgeist, vielleicht auch eine gewisse Bildung sind für diesen Priester eines ländlichen Heiligtums zu konstatieren. Von Vertretern der Stadt Sardeis ist nicht die Rede. Es hat den Anschein, daß der Proconsul von der unbeschränkten Handlungsfähigkeit der beiden Vertreter der Landgemeinde ausgeht. Das könnte bedeuten, daß Sardeis bei dieser Marktrechtsverleihung nicht mehr als das von dem Proconsul eingeräumte Widerspruchsrecht blieb. Allerdings können wir nicht ausschließen, daß es vor dieser Rechtsgewährung durch den Proconsul irgendwelche, zumindest informelle Kontakte mit dem Zentralort, also mit Sardeis, gegeben hat. Wie auch immer, es ist bemerkenswert, daß Bevollmächtigte einer abhängigen Landgemeinde vom Proconsul als die eigentlichen Privilegienempfänger behandelt werden.

Die Arillenoi verfügten, wie aus dem Text der Stele hervorgeht, über die lateinische Fassung des Privilegientextes; anscheinend nahmen die beiden dörflichen Gesandten die Originalurkunde mit in ihr Heimatdorf. Sie war also nicht für das Archiv des Zentralortes bestimmt. Wäre die Urkunde, wie im Falle der Marktprivilegien für Mandragoreis, im Archiv der übergeordneten Stadt deponiert worden, so hätte die Landgemeinde sich kaum um den lateinischen Text bemüht, sondern mit einer Kopie der griechischen Übersetzung begnügt. Trifft diese Interpretation das Richtige, so ergibt sich daraus ein weitgehend autonomes Agieren der Landgemeinde auch bei der Archivierung der Privilegien, die somit in erster Linie als Rechtsgewährung für das Dorf und nicht für den Zentralort verstanden wurden.

Die Publikation des lateinischen Textes auf der Stele ist bemerkenswert; kaum einer unter den Dorfbewohnern dürfte dieser Sprache mächtig gewesen sein. Es geht also mit Sicherheit nicht um eine größere Publizität des Textes. Ehrerbietung und Dank gegenüber dem Gewährer des neuen Rechts, möglicherweise auch Stolz über die direkten Kontakte mit der lateinischsprachigen Reichsadministration, vielleicht auch das etwas naive Bestreben, dem gewährten Privileg durch die Publikation in Griechisch und Latein größere Rechtssicherheit und Verbindlichkeit zu geben, mögen Gründe für die zweisprachige Publikation sein.

Im Dorf der Arillenoi trug die Marktrechtsverleihung dem Initiator großen Beifall ein; er wurde öffentlich geehrt: Jedenfalls sprach die Dorfversammlung 48 ihm und seiner Frau einen goldenen Kranz zu, den auch ihre Nachkommen tragen durften. Aus der Ehrung ist abzuleiten, daß die Gewinnung des Marktrechtes im Dorf beträchtliche Resonanz fand. Bemerkenswert bei diesen beiden von der Dorfversammlung der Arillenoi ge- und verfaßten Ehrenbeschlüssen, die in dem freigebliebenen Raum der Stele nachgetragen wurden, sind die schlechte Ortho-

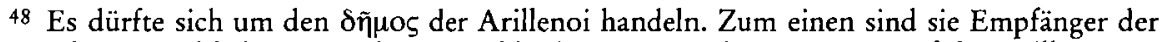
Privilegien und haben Grund zur Dankbarkeit, zum anderen ist im Dorf der Arillenoi ein nicht weiter spezifizierter $\delta \tilde{\eta} \mu \circ \zeta$ mit der dort zusammentretenden Gemeindeversammlung zu identifizieren. 
graphie und wenigstens ein schwerer Verstoß gegen die Regeln der griechischen Grammatik: Der Genetiv von үévos lautet in beiden Ehrenbeschlüssen der Dorf-

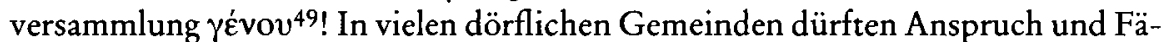
higkeit, in städtischen Formen zu agieren, deutlich auseinandergeklafft haben.

Der Stolz einer Dorfgemeinde, einen eigenen Markt zu besitzen, ist auch in der Inschrift von Mandragoreis gespiegelt. Der Markt wurde mit einer Feier eingeweiht, zu der einer der legati des Proconsuls der Provinz Asia eingeladen wurde

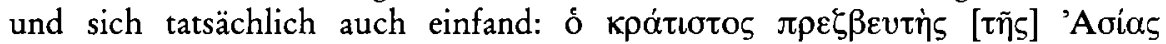

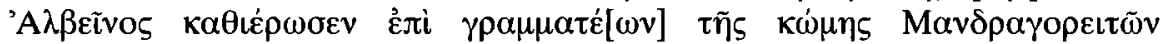

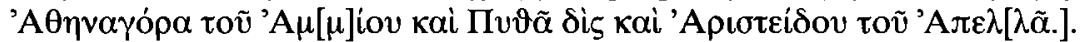

Schließlich gibt auch das archäologische Monument Marktstele einige Anhaltspunkte für das mit seiner Anfertigung und Aufstellung verbundene dörfliche Selbstverständnis. Zumindest in einigen Fällen legten die Empfänger derartiger Privilegien großen Wert auf eine dauerhafte und würdige Publikation ihrer neuen Rechte.

Im Falle der Pyliten geht der Wunsch nach Aufzeichnung der dörflichen Privilegien vom Rat der übergeordneten Stadt aus und wird dann vom Patron des

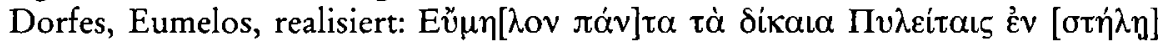

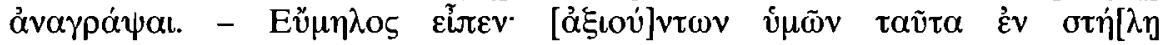

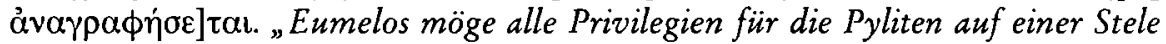
aufzeichnen!" Eumelos sagte: ,Wenn Ibr so wollt, werden diese auf einer Stele aufgezeichnet. " Der Rat der Stadt, der sich beim Proconsul für die Rechtsgewährung eingesetzt hatte, freute sich über die Privilegierung des Dorfes und betrieb nachdrücklich die Publikation, nicht zuletzt um damit seine Rolle als großer Wohltäter des Dorfes der Pyliten herauszustellen. Die Privilegien für die Pyliten und die mit ihrer Gewährung verbundenen Dokumente und Protokolle wurden auf minde-

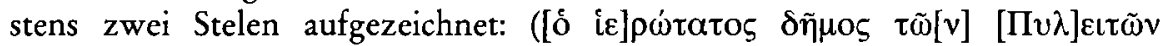

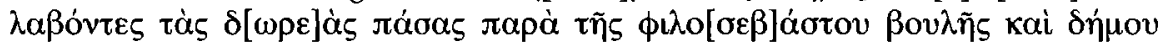

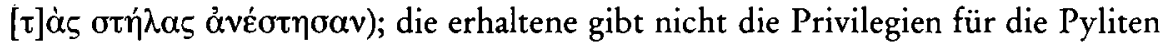
wieder, sondern berichtet nur davon, wie diese Privilegien vom Proconsul bestätigt wurden und wie diese Bestätigung im Rat der übergeordneten Stadt aufgenommen wurde. Die Aufzeichnung wurde als wichtiger Akt angesehen. Ungewöhnlich ist, daß der Steinmetz, ein Mann aus Halikarnass, der der Qualität seiner Schrift nach zu urteilen nur bescheidene Fähigkeiten besaß, eigens genannt ist. Anscheinend war es am Ende des 3. Jahrhunderts n. Chr. in einer ländlichen Region des unteren Mäandertals schwierig, einen Mann zu finden, der solche Aufträge ausführen konnte; das Beharren auf eine inschriftliche Form der Publizität besitzt bei einer solchen Situation schon einiges an Aussagekraft.

Im Falle der Privilegien für die Arillenoi, Mandragoreis und Pyliten wurden die Privilegien auf Stelen aufgezeichnet. Im Falle des Privilegs für Tetrapyrgia spricht einiges dafür, daß es ebenfalls auf einer Stele aufgezeichnet war. Wahrscheinlich standen sie auf der Agora, wo der Markt abgehalten wurde. Dort kündeten sie von

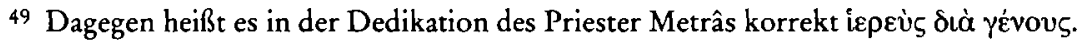


dem Marktrecht des betreffenden Dorfes, waren also ein Marktmal. Die Form des Inschriftenträgers ist durchaus von Bedeutung. Wichtige Privilegien wurden in Städten oft auf großen Stelen aufgezeichnet. Wir dürfen davon ausgehen, daß selbst Leseunkundige in einem bestimmten baulichen oder räumlichen Kontext mit Stelen die Verleihung wichtiger Privilegien verbanden; dazu trugen nicht zuletzt die Münzen der Städte bei: Mit der Wiedergabe von Stelen auf den städtischen Münzen wurde auf die Verleihung von Privilegien hingewiesen. Münzen von Kremna, Seleukeia am Kalykadnos, Termessos und Side bilden derartige Stelen im Zusammenhang mit dem Erhalt von wichtigen Privilegien ab. In einigen Fällen ist in die Stelen der Münzbilder zur Verdeutlichung noch das Wort $\delta \omega \rho \varepsilon \alpha ́$ hineingeschrieben, d.h. Privileg5. Landgemeinden bedienten sich einer städtischen Gepflogenheit, wenn sie ihre Privilegien auf Stelen publizierten und damit ihre rechtliche Ausstattung unterstrichen.

Im Falle der Marktprivilegierung für die Arillenoi zeigt sich, daß es in einer Landgemeinde Männer gab, die sich direkt an den Leiter der römischen Provinzadministration wandten, bei ihm vorstellig und als Rechtsvertreter akzeptiert wurden. Aus dem Marktprivileg für Mandragoreis können wir konstatieren, daß die römische Administration den Menschen auf dem Lande ihre Achtung nicht versagte, wenn etwa zur feierlichen Einweihung des Dekadenmarktes ein Legat des Proconsuls erschien und die Einweihung vollzog. Die Feststellung, die Roger S. Bagnall über das Verhältnis von Staat und Dorf im kaiserzeitlichen Ägypten getroffen hat: „The state's interest in the village was largely one of collecting taxes, extracting labor, and preventing disturbance " 51 , möchte ich nicht grundsätzlich in Frage stellen, doch zeigt sich, daß die Beziehungen von römischer Provinzverwaltung und lokalen Administrationskörperschaften - zumal in anderen Regionen des Römischen Reiches - nicht notwendigerweise und nicht ausschließlich diese Formen annehmen mußten, sondern komplexer und vielgestaltiger sein konnten.

\section{Dörfer und ihre Patrone}

Die Etablierung von ländlichen Märkten war keine Angelegenheit, die ausschließlich die römische Provinzadministration, die Dörfer und ihre Zentralorte betraf. Von Bedeutung waren sehr oft Persönlichkeiten, die in diesen Dörfern, wenn nicht sogar über diese Dörfer große Macht hatten. Der Einfluß dieser Leute beruhte anscheinend auf Grundbesitz, den sie in den Dorffluren hatten. Nicht aus-

$50 \mathrm{Vgl}$. die Inschrift der Pyliten, wo die Privilegien genau mit diesem Terminus bezeichnet werden. Zu den Inschriftenstelen auf Münzen vgl. Peter Weiß, Zu Münzprägungen mit den

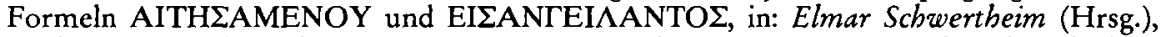
Studien zum antiken Kleinasien II (Asia Minor Studien 8, Bonn 1992) 178f. (Side, Seleukeia am Kalykadnos, Kremna, Termessos, Ankyra Sidera).

51 Roger S. Bagnall, Official and Private Violence in Roman Egypt, in: BASP 26 (1989) 201216, h. 204. 
zuschließen ist, daß in einigen Fällen Dörfer direkt auf dem Grund und Boden eines großen Gutes standen. Diese einflußreichen Personen übten eine Art von Patronat über die Landgemeinden ihrer Einflußsphäre aus; sie werden unter

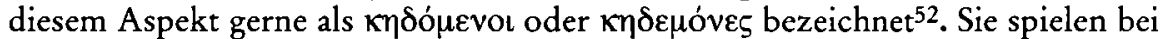
allen hier zu diskutierenden Marktprivilegierungen eine Rolle, wenn auch in unterschiedlicher Art und Weise.

Bei der Marktrechtsverleihung an die Arillenoi scheint die Landgemeinde völlig unabhängig von einem solchen Patron durch seine Vertreter zu agieren. Von der Existenz eines mächtigen und einflußreichen Landbesitzers erfahren wir zunächst nichts. Er kommt erst ins Spiel, als das Dorf der Arillenoi mit einem Problem konfrontiert wird, das die führenden Persönlichkeiten des Dorfes anscheinend nicht selber lösen konnten: Während der Herrschaft des Antoninus Pius unternahm die Stadt Sardeis den Versuch, beim Kaiser ergänzende Privilegien, vermutlich insbesondere die Steuerfreiheit, für Dörfer auf ihrem Territorium zu gewinnen, die wie die Arillenoi Jahrmärkte abhalten durften. Offensichtlich waren die Arillenoi, aus welchen Gründen auch immer, für eine solche Rechtserweiterung nicht vorgesehen. In dieser Situation schalteten sie einen Fürsprecher ein. Aus einem auf der Marktstele nachgetragenen Dokument erfahren wir, daß ein Mann namens Asinius Rufus in der Gemeinde der Arillenoi von großem Einfluß war; er hielt sich vermutlich in der Fremde auf. Vieles spricht dafür, daß es sich bei Asinius Rufus um einen in den Senatorenstand aufgestiegenen Sproß einer alten sardianischen Honoratiorenfamilie handelt. Dieser Asinius Rufus sagt von sich, daß das Dorf der Arillenoi schon seit Generationen in einem engen Verhältnis zu ihm steht

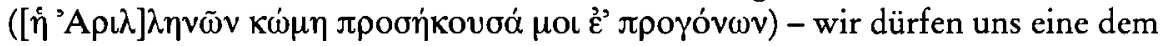
Patronat ähnliche Beziehung vorstellen, die anscheinend auf bedeutendem Grundbesitz beruht. Er machte seinen ganzen Einfluß bei dem Archontenkollegium von Sardeis geltend, damit die Arillenoi mit den anderen Dörfern gleichgestellt und auch für sie ergänzende Privilegien für ihren Jahrmarkt beantragt wür-

$52 \mathrm{Zu}$ dem häufig verwendeten, sehr verschiedene Verhältnisse bezeichnenden Wort vgl. Louis Robert, in: A. Dupont-Sommer, ders., La déesse de Hiérapolis Castabala (Cilicie) (Paris

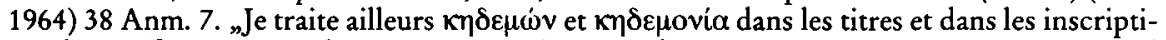
ons honorifiques.“; Hugh J. Mason, Greek Terms for Roman Institutions. A Lexicon and Analysis (Toronto 1974) 60f.; André Balland, Fouilles de Xanthos VII. Inscriptions d'époque impériale du Létôon (Paris 1981) 87 Anm. 74: „l'acception est très étendue, recouvrant à la fois tutor et curator (Dig. 19,2,49-Modest.); pour des emplois plus larges et moins juridiques du mot, L. Robert, OMS IV, p. 283. “; Ivars Avotins, On the Greek of the Code of Justinian, A Supplement ot Liddell-Scott-Jones together with Observations on the Influence of Latin on Legal Greek (Hildesheim, Zürich, New York 1989; zuerst in: ders., On the Greek Vocabulary of the Digest, in: Glotta 60 [1982] 247-280, bes. 262f.) $85 \mathrm{ff} .:$ „To sum up, $\kappa \eta \delta \varepsilon \mu \omega \dot{v}$ and $\kappa \eta \delta \varepsilon \mu o v i \alpha$ are used both as more general terms, subsuming under them both the tutorship and the curatorship, and in the narrower meanings é $\pi i \tau \rho \circ \pi \circ$ (tutor) and curator." Und schließlich Marianne Blume, A propos de P. Oxy. I, 41. Des acclamations en l'honneur d'un prytane confrontées aux témoignages épigraphiques du reste de l'empire, in: Lucia Criscuolo, Giovanni Geraci (Hrsg.), Egitto e storia antica dall'Ellenismo all'età araba. Bilancio di un confronto. Atti del Colloquio Internazionale Bologna, 31 agosto - 2 settembre 1987 (Bologna 1989) 271-290, bes. 283 ff. 
den. Wahrscheinlich hatte Asinius Rufus mit seiner Intervention bei den sardianischen Archonten Erfolg, so daß sein Schreiben für wert befunden wurde, auf der Marktstele aufgezeichnet zu werden 53 .

Ungefähr siebzig Jahre später, als Mandragoreis auf dem Territorium von Magnesia am Mäander seine Marktrechte erhalten soll, traten nicht Vertreter des Dorfes (etwa die drei eponymen Grammateis) vor dem Proconsul auf, sondern

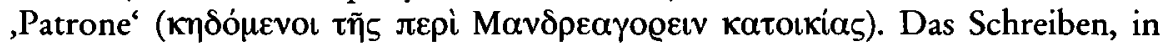
dem der Proconsul um Marktrechte gebeten wird, könnte von einem dieser Fürsprecher stammen ${ }^{54}$.

Im Falle der Privilegierung des Marktes von Tetrapyrgia aus dem Jahr 254 hat ein gewisser Domitius Rufus, dessen Vater Asiarch war, einen Antrag auf die Gewährung eines Markttages pro Monat gestellt. Dieses wird dem - vermutlich maionischen - Honoratioren vom Proconsul in einem persönlichen Schreiben, nicht in einem Edikt wie im Falle der Arillenoi und Mandragoriten, gewährt. Der Proconsul bezeichnet diese Privilegierung als Ehrung für den Honoratioren und nicht als direkte Privilegierung des Dorfes Tetrapyrgia. Die Gewährung an den Honoratioren wird nicht nur mit dessen persönlichen Vorzügen begründet, sondern auch damit, daß er in dem Dorf die Verehrung für die Götter etabliert hat:

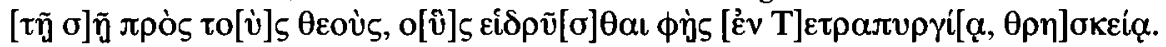
Natürlich geht es um heidnische Götter, vermutlich auch um den Kaiserkult, wie wir bei einem Sohn eines Kaiserpriesters erwarten können. Wahrscheinlich sollte der zu etablierende Markt unter dem Schutz dieser Gottheiten stehen, fand vielleicht in der Nähe eines neuerrichteten Heiligtums stand. Hintergrund für den hier besonders herausgestellten Zusammenhang zwischen Privilegiengewährung und Förderung des heidnischen Kultes dürften die religiösen Auseinandersetzungen der Zeit sein; die Versuche des Decius, die Verehrung der heidnischen Götter, wenn nötig, mit $Z$ wang durchzusetzen, lag noch nicht lange zurück; die bald nach 254 erneut einsetzenden Christenverfolgungen zeigen, daß keine wirkliche religiöse Entspannung eingetreten war ${ }^{55}$. Wie sehr bei der Privilegienvergabe an Dörfer in den nächsten Jahrzehnten religiöse Faktoren eine Rolle spielen konnten, zeigt auch die Stadterhebung von Orkistos durch Konstantin. In seinem Brief greift er als Begründung für die Privilegierung ausdrücklich das Argument der Orkistanoi auf, die Einwohner des Ortes seien Anhänger des Christentums: qui-

53 Zu den Einzelheiten vgl. Eck, Nollé, Brief (Anm. 30).

54 Mitchell, Anatolia (Anm. 4) 184 Anm. 174 schließt hingegen aus den Anredeformen

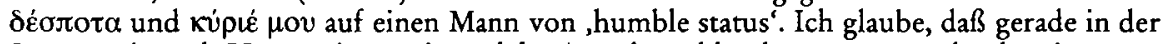
Severerzeit auch Honoratioren eine solche Anrede wählen konnten; so reden bereits unter Antoninus Pius ritterliche Procuratoren von Mauretanien die Statthalter von Numidien mit ,domine' an (ILS 5795); für den Gebrauch dieser Anrede vgl. die noch immer sehr nützliche Zusammenstellung von Martin Bang, Über den Gebrauch der Anrede domine im gemeinen Leben, in: Ludwig Friedländer, Darstellungen aus der Sittengeschichte Roms in der Zeit von Augustus bis zum Ausgang der Antonine 4 (Leipzig 1921) 82-88.

$55 \mathrm{Zu}$ der Christenverfolgung des Valerian vgl. z. B. Karl-Heinz Scbwarte, Die Christengesetze Valerians, in: W. Eck (Hrsg.), Religion und Gesellschaft in der römischen Kaiserzeit (Köln, Wien 1989) 103-163. 
bus omnibus quasi quidam cumulus accedit quod omnes [i]bidem sectatores sanctissimae religionis babitare dicantur ${ }^{56}$.

Religiöse Gründe scheinen auch bei dem Engagement des $\kappa \eta \delta \varepsilon \mu \omega ́ v$ der Pyliten,

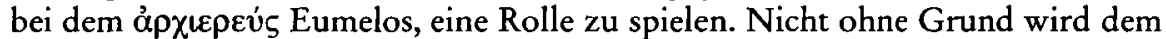
Gott Dionysos akklamiert, dessen Kult vielleicht in dem Dorf der Pyliten eine wichtige Rolle spielte ${ }^{57}$.

Einflußreiche und vermögende Großgrundbesitzer, die entweder zu den Honoratioren der übergeordneten Stadt gehörten oder bereits in die Reichsaristokratie aufgestiegen waren, erscheinen in den Marktinschriften in der Rolle von Interessensvertretern der Dörfer bei Verhandlungen mit der sie administrierenden Polis wie auch der römischen Administration. Es ist nicht völlig klar, ob die Dörfer auf deren Grundbesitz liegen, also zumindest faktisch diesen Honoratioren gehören und es sich somit um die Etablierung eines ,Domänenmarktes' handelt, wie das bei einigen der nordafrikanischen Marktrechtsverleihungen der Fall ist. Jedenfalls ist ein solcher Schluß in keiner Weise zwingend; für die Arillenoi ist eine derartige Unterstellung aus verschiedenen Gründen eher unwahrscheinlich, für die Tetrapyrgiten ist sie nicht auszuschließen. Es liegt in der Natur der Sache, daß die einflußreichen Persönlichkeiten nicht nur die Interessen von Schutzbefohlenen vertraten, sondern auch eigene Absichten bei der Einrichtung von Märkten hatten.

\section{Dörfer und die sie administrierende Stadt}

Wie stellten sich die Städte zu dem Bestreben der Dörfer, auf ihrem Territorium Marktrechte zu gewinnen? Förderten oder behinderten sie ländliche Märkte? Aufgrund der erhaltenen Inschriften ist nur schwer zu argumentieren, da wir durch sie nur Einblick in jene Fälle bekommen, in denen die Errichtung von Märkten durch die übergeordneten Städte nicht verhindert wurde.

In jedem Fall wurde von der römischen Administration bei der Gewährung von Marktrechten auf die Märkte der übergeordneten Städte (und anderer Dörfer in der Gegend) Rücksicht genommen. Der Antragsteller auf Marktrecht für Mandragoreis, der diesen Grundsatz anscheinend genau kennt, weist ausdrücklich darauf hin, daß der beantragte ländliche Markt weder der Stadt Magnesia am Mäander noch dem kaiserlichen Fiscus Schaden bringe. Der Proconsul räumt bei seiner Gewährung des Jahrmarktes für die Arillenoi eine Einspruchsfrist von

56 Chastagnol, Orcistus (Anm. 26) 386 Z. $39 \mathrm{ff}$. mit den Kommentaren $409 \mathrm{ff}$.

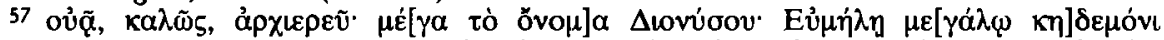

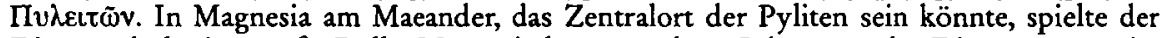
Dionysoskult eine große Rolle: Magnesia beanspruchte, Geburtsort des Dionysos zu sein; vgl. Albert Henrichs, Greek Maenadism from Olympias to Messalina, in: HSCP 82 (1978) 121-160, bes. 123-137: Theban Maenads in Magnesia ad Maeandrum; Louis Robert, Documents d'Asie Mineure XVIII. Fleuves et cultes d'Aizanoi, in: BCH 105 (1981) 331-360, bes. 355-360. 


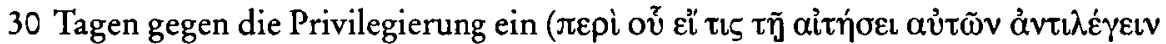

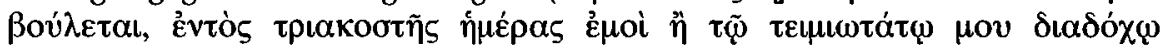

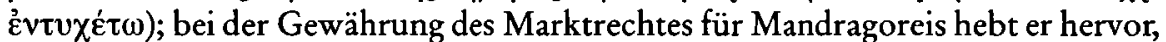
$\mathrm{da}$ die Patrone der Stadt versichert haben, daß sie mit ihrem Antrag niemandem

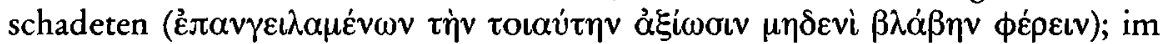
Falle der Marktrechtsverleihung an Domitius Rufus für Tetrapyrgia scheint eine ähnliche Versicherung ergangen zu sein, auf die der Proconsul in seinem Brief verweist: Keines der Dörfer auf dem Territorium von Maionia, die schon zuvor Marktprivilegien bekommen hatten, halte an diesem Tag einen Markt ab $(\mu[\eta] \delta \varepsilon \mu \tilde{\alpha} \varsigma[\pi \alpha \sigma \tilde{\omega} v] \tau \tilde{\omega} v \kappa \alpha \tau[\dot{\alpha}] \tau \dot{\eta} v \operatorname{M\alpha } \sigma v[i] \alpha v \phi \theta[\alpha v] \operatorname{lov\sigma \tilde {\omega }v}[\kappa \omega \mu \tilde{\omega} v \tau] \alpha \dot{v} \tau \eta$

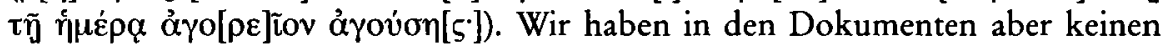
Hinweis darauf, daß ländliche Märkte nur nach ausdrücklicher Zustimmung der Stadt verliehen wurden; mit anderen Worten, nichts deutet darauf hin, daß die Dörfer zuerst die Zustimmung der sie administrierenden Städte vorlegen mußten. Anscheinend sicherte die römische Provinzadministration den Städten lediglich ein Einspruchsrecht.

Weiteres über das Verhältnis von Dorf und Stadt erfahren wir aus den Archivvermerken: Die Urkunde über die Verleihung der Marktrechte wurde im Falle des Marktes für Mandragoreis, möglicherweise von einem der dörflichen Patrone, zunächst im Archiv der übergeordneten Stadt archiviert. Das Dorf Mandragoreis mußte sich eine Abschrift im Archiv von Magnesia besorgen. Im Falle des Marktrechts für Tetrapyrgia erhält das Dorf, anscheinend von seinem Patron Domitius Rufus, den an ihn gerichteten Originalbrief mit der Marktrechtsgewährung. Wenn wir das stark zerstörte Ende der Inschrift richtig deuten, so wird der Brief mit Abschriften aber schließlich im Archiv der übergeordneten Stadt deponiert. Im Falle der Privilegierung der Pyliten kommt der Brief des Proconsuls zunächst an den Rat der übergeordneten Stadt, wo er begeistert aufgenommen wird. Das pronconsularische Schreiben wird auf Antrag eines Ratsmitgliedes im Archiv der Stadt niedergelegt. Die Deponierung im städtischen Zentralarchiv dürfte vielleicht mit der Registrierung des Privilegs und damit auch einer gewünschten Berücksichtigung durch die übergeordnete Stadt, vor allem aber mit einer sicheren Archivierung zu tun haben.

Im Falle der Privilegien für die Pyliten setzt die übergeordnete Stadt sich für die Interessen ihres Dorfes ein. Dort heißt es im Brief des Proconsuls ausdrücklich, daß der Statthalter die Dankbarkeit und das Engagement der städtischen Gremien für die ländliche Gemeinde lobt. Bei einem solchen Einvernehmen haben wir das Wirken der Patrone in Betracht zu ziehen, die in den Städten als Honoratioren oftmals eine wichtige Rolle in den städtischen Gremien spielten und Interessenskonflikte zwischen Dorf und Stadt zu verhindern halfen und mit der Privilegierung einzelner Dörfer auch ihren eigenen Interessen dienten.

Spannungen zwischen Stadt und Dorf werden anscheinend im Falle der Arillenoi sichtbar. Hier scheint es zu einem gewissen Konflikt bei der Erweiterung der Marktrechte für die Arillenoi gekommen zu sein. Wir müssen unterstellen, daß die sardianischen Behörden sich nicht für eine weitere Privilegierung des Jahrmarktes 
der Arillenoi einsetzen wollten. Die Hintergründe dafür kennen wir nicht. Es dürfte aber eine Rolle gespielt haben, daß der Patron der Arillenoi nicht in Sardeis weilte und sich so nicht unmittelbar in den städtischen Gremien und bei den Magistraten für seine Schutzbefohlenen einsetzen konnte.

Man kann daran zweifeln, daß dieses Bild von einem recht ungetrübten Verhältnis zwischen Dorf und Stadt die ganze Wirklichkeit wiedergibt. Eine Reihe von Überlegungen und Interessen hätten Städte durchaus dazu bringen können, sich gegen die Etablierung von ländlichen Märkten zu wehren. Tatsächlich wissen wir aus Plinius von einem solchen Fall: Im Jahre 105 verwahrten sich die Bewohner der Stadt Vicetia dagegen, daß ein gewisser L. Bellicius Sollers auf seinem Gut durch Beschluß des Senates Markttage etablierte ${ }^{58}$. Märkte konnten die städtische Wirtschaft und die Interessen bestimmter Gruppen in der Stadt beeinträchtigen; Märkte stärkten zweifellos das Selbstbewußtsein der Dörfer und damit in bestimmten Fällen auch zentrifugale Tendenzen. Allerdings scheint mir das Bild von Pierre Debord zu einseitig: Man wird feststellen, daß wäbrend der gesamten Kaiserzeit der Wille der Dörfer, sich von den Städten zu trennen, die sie kontrollierten und wabrscheinlich auch wirtschaftlich ausbeuteten, klar anf der Hand zu liegen scheint. Und wenig später: Die ländliche Bevölkerung hat, in ständig steigender Zabl, eine totale Unabbängigkeit gewonnen ${ }^{59}$.

Die Gefahr, daß ein Dorf sich aufgrund eines Marktes so entwickelte, daß es sich aus dem Stadtverband löste, dürfte recht gering gewesen sein. Wichtig bei solchen Überlegungen erscheint es mir, zwischen monatlich ein- oder mehrmals abgehaltenen Märkten und Jahrmärkten zu unterscheiden. Jahrmärkte dürften niemals zu einer wirklichen Konkurrenz für die Wirtschaftsinteressen einer Stadt geworden sein. Sie konnten auch keine nachhaltige Veränderung der wirtschaftlichen Situation eines Dorfes erreichen. Sich ihrer Etablierung entgegenzustellen hätte einen Affront gegen die ländlichen Religiosität und die Feste der Bauern bedeutet. Wochenmärkte waren hingegen problematischer; eine Ausstattung mehrerer Dörfer mit ihnen führte jedoch dazu, daß sich kein für die Stadt konkurrierendes Zentrum entwickeln konnte. Die Ausstattung eines Dorfes mit monatlichem oder häufigerem Marktrecht dürfte eher der Versorgung der ländlichen Bevölkerung gedient haben. Ein gut ausgebautes Marktnetz mit Ausrichtung auf die Stadt erleichterte vielen Händlern den Weg in die Stadt und konnte somit einer Stadt insgesamt größere Einnahmen verschaffen.

58 Vgl. dazu de Ligt, Fairs and Markets (Anm. 36) 202-208.

59 Pierre Debord, Populations rurales de l'Anatolie gréco-romaine, Atti (Centro ricerche e documentazione sull'antichità classica RDAC) $8(1976-1977) 43-68$, bes. 61 . 


\section{Ein Ausblick}

In der Provinz Asia gab es in der Römischen Kaiserzeit mancherorts in der Landbevölkerung engagierte Persönlichkeiten, die bestrebt waren, die Entwicklung ihrer Dörfer wirtschaftlich und administrativ voranzutreiben, und die sich dabei sogar an die Spitzen der römischen Provinzverwaltung wandten. Marktrechte spielten als substantielle wie auch prestigeträchtige Privilegien von Landgemeinden eine wichtige Rolle. Ländliche Honoratioren der Dorfgemeinden konnten zu ihrer Gewinnung jene Spielräume nutzen, die ihnen gemeinsames Agieren bald mit der Administration des Zentralorts, bald mit einem an den lokalen Verhältnissen interessierten Patron eröffneten. Die Anbindung der angestrebten Marktrechte an religiöse Einrichtungen wie Feste und Heiligtümer verlieh dem Wunsch der Landbevölkerung besonderen, möglicherweise manchmal ausschlaggebenden Nachdruck. Das komplizierte Beziehungsgeflecht von Stadt und Land, von Zentralort und abhängiger Landgemeinde ${ }^{60}$, bot, anscheinend mehr situativ als rechtlich abgesichert ${ }^{61}$, die Möglichkeit einer begrenzten lokalen Autonomie, die auch bei den Spitzen der römischen Administration eine gewisse Anerkennung finden konnte. Wir dürfen uns die Spielräume allerdings nicht allzu groß vorstellen; gegen die Interessen des Zentralortes und gegen dessen erklärten Willen dürften Privilegien für ländliche Siedlungen bei der römischen Administration kaum zu erreichen gewesen sein.

Die Versuchung liegt nahe, von den hier diskutierten vier Texten aus weitergehende Fragen zu stellen:

Wurde die Etablierung von ländlichen Märkten in der Zeit Hadrians durch das Bestreben dieses Kaisers gefördert, im römischen Kleinasien die Urbanisierung voranzutreiben? Gehen also nicht nur Stadtgründungen und Stadterhebungen im ländlichen Raum, wie etwa in Mysien, auf diesen Kaiser zurück, sondern ermutigten Hadrians Reisen und seine Kontakte mit der Landbevölkerung auch deren Bestrebungen, urbaner zu werden und sich um eine Verbesserung der ländlichen Infrastruktur zu bemühen? Eröffnete die kaiserliche Politik auf diese Weise neuen Spielraum für lokale Autonomiebestrebungen?

Hat die Vergabe neuer Marktrechte unter Septimius Severus mit dem unbestreitbaren Engagement dieses Kaisers für die Landbevölkerung zu tun? Bestätigen Marktrechtsverleihungen der Severerzeit Michael Rostovtzeff, der meint, „the policy of Septimius Severus towards the humble was policy of protection and concession" ${ }^{\circ} 62$ ?

60 Ein meiner Meinung nach recht zutreffendes Bild zeichnet Kolb, Stadt (Anm. 11) 238260, bes. 256: „Im allgemeinen dürften die sozialen Kontakte zwischen Stadt und Land im römischen Reich relativ eng gewesen sein." Dagegen halte ich das von Mitchell, Anatolia (Anm. 4) gezeichnete Bild einer, world apart' für recht problematisch.

61 Vgl. die richtige Feststellung von Kolb, Stadt (Anm. 11) 257: „Ebensowenig wie Rechtsverhältnisse ausschlaggebend für sozialen Aufstieg waren, haben sie die Beziehungen zwischen Stadt und Land entscheidend beeinflußt."

$62 \mathrm{Vgl}$. Michael Rostovtzeff, The Social and Economic History of the Roman Empire (Ox- 
Spiegelt die anscheinend steigende Bedeutung einflußreicher Patrone bei der Gewinnung von Marktrechten deren zunehmende Macht auf dem Lande, vielleicht sogar die Anfänge der Schaffung exemter Territorien auf den Stadtgebieten $^{63}$ ? Verliert der Zentralort allmählich seine faktische Verfügungsgewalt über seine administrativen Untereinheiten an mächtige Grundherren? Ist es vielleicht so, daß die Dörfer, die über einige Zeit eine gewisse Autonomie durch den Interessengegensatz von Zentralort und mächtigen Patronen gewinnen konnten, am Ende eine Herrschaft gegen eine andere eintauschten?

Haben die im Laufe des 3. Jahrhunderts und noch zu Beginn des 4. Jahrhunderts intensiv ausgetragenen religiösen Auseinandersetzungen neue Spielräume für lokale Autonomie eröffnet? Haben die Anhänger der alten heidnischen Kulte versucht, die Landbevölkerung durch Privilegiengewährungen für ländliche Märkte, die im Zusammenhang mit Heiligtümern standen, an die tradierten Kulte und auch an die althergebrachte Ordnung zu binden?

Die Überlieferung reicht, so meine ich, jetzt noch nicht aus, um diese Fragen verläßlich zu beantworten. Es bleibt nur zu hoffen, daß weitere Surveys in Kleinasien Dokumente ans Tageslicht bringen, die Antworten auf einige dieser Fragen erlauben.

ford 21957) 406; vgl. auch Naphthali Lewis, The Humane Legislation of Septimius Severus, in: Historia 45 (1996) 104-113, der 112 zu dem Schluß kommt: „We witness, too, the protection of the defenceless country-dwellers against the more powerful and influential townspeople. " Die severische Politik bringt z. B. auch Scheidel, Dokument (Anm. 11) 156f. bei seinen Überlegungen zu den Petitionen von Dorfleuten direkt an den Kaiser ins Spiel.

$63 \mathrm{Vgl}$. dazu etwa den von Herennius Modestinus verkündeten Grundsatz (Dig. 50, 1, 35) „der Herauslösung aller großen Grundbesitzer aus den Bindungen an die Stadt" (Werner Eck, in: Thomas Drew-Bear, Peter Herrmann, ders., Sacrae Litterae, in: Chiron 7 [1977] 355383). Zu der allgemeinen Tendenz Hagen Fischer, Zu Problemen von Stadt und Stadtentwicklung im Römischen Reich während des 3. Jahrhunderts, in: Klaus-Peter Jobne (Hrsg.), Gesellschaft und Wirtschaft des Römischen Reiches im 3. Jahrhundert (Berlin 1993) 135-185, bes. 141: „Da jedoch von verschiedenen antiken Autoren seit Ende des 1. Jahrhunderts gefordert wird, Kolonen ganz allgemein statt mit Geldpacht mit Naturalpacht zu belegen, ist anzunehmen, daß weit vor dem 3. Jahrhundert ein allmählicher Prozeß der Loslösung der Kolonen von der Stadt beginnt.“ Denn „bei dieser Pachtform stand nicht mehr der städtische Markt als Vermittler zwischen Pächter und Verpächter“. 
